\title{
Architecture of Data Access Based on Power Dispatch Control Cloud Zhou Xiaoming ${ }^{1, a^{*}}$, Tao Lei ${ }^{1, b}$, Li Dapeng ${ }^{1, \mathrm{c}}$ and Xie Tao ${ }^{1, \mathrm{~d}}$ \\ ${ }^{1}$ China Electric Power Research Institute, Haidian District, Beijing 100192, China \\ aemail: zhouxiaoming@epri.sgcc.com.cn, bemail: taolei@epri.sgcc.com.cn,

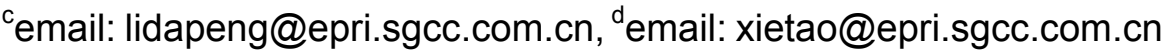

\begin{abstract}
Keywords: Power Dispatch Control Cloud; Client-Proxy-Proxy-Server; Data Proxy Service; Cloud Center
\end{abstract}

\begin{abstract}
The data synchronization between all the regional dispatching system under the dispatch control center and power dispatch control cloud has been realized. The data information from each region such as real-time data, manipulate data, model data and graph data firstly is sent to the communication server for this region. Besides, the communication server sends this data to the dispatch control cloud center after some processing. In order to obtain the resource from cloud center, the workstations in each region have to access cloud center remotely. However, most process in man-machine remote access adopts client/server architecture, which affects the security of data access. To solve this problem and provide data proxy service in cloud center, a new architecture of data access based on power dispatch control cloud is proposed in this paper.The architecture of data access is a client-proxy-proxy-server architecture, which can not only ensure remote workstation accessing its own resource from the cloud center, but also ensure it obtaining resource of other regions from the cloud center with not switching the platform environment of smart grid dispatching and control system.
\end{abstract}

\section{Introduction}

Electric power integrated dispatch control system based on Clouding Computing Technology (Power Dispatch Control Cloud) was built into two cloud centers in two regions respectively. These two cloud centers form a new pattern which is identical logically, and dispersed physically. These two cloud centers make up the primary and backup system physically and provide logically unified service to users ${ }^{[1-2]}$. Certainly some service such as data service and network analysis are provided for all areas belonging to dispatching control center. In the implementation of Technology, based on distributed, virtualization and parallel computing technology, details of development such as structure, function design and implementation plan of system were considered in advance to ensure high reliability of integrated dispatch control system ${ }^{[3-6]}$. Based on our system, construction investment of integrated control system is saved and development of dispatch control system is supported in basic technology ${ }^{[7]}$.

First of all, the real-time data is stored and managed in data layer and provides more support of running data for the whole network analysis, big data analysis and data mining, etc. ${ }^{[8-11]}$. Secondly, real-time data dispatch business is monitored in the business layer. Finally, in the system layer, we realize grid operation, monitoring function and the interfaces of all region's dispatching institutions for unified human computer technology ${ }^{[12]}$.

The construction ideas of the dispatch control cloud is shown in figure 1: firstly, the data information from each region such as real-time data, manipulate data, model data and graph data firstly is sent to the communication server which is corresponding to location of the cloud. And then information is transmitted to the cloud after communication server. In the next, remote workstation of regions can visit various business modules in cloud on communication server deployed in cloud. At last, grid operation monitoring and centralized monitoring of substation at every level have been achieved in the virtual cloud. 


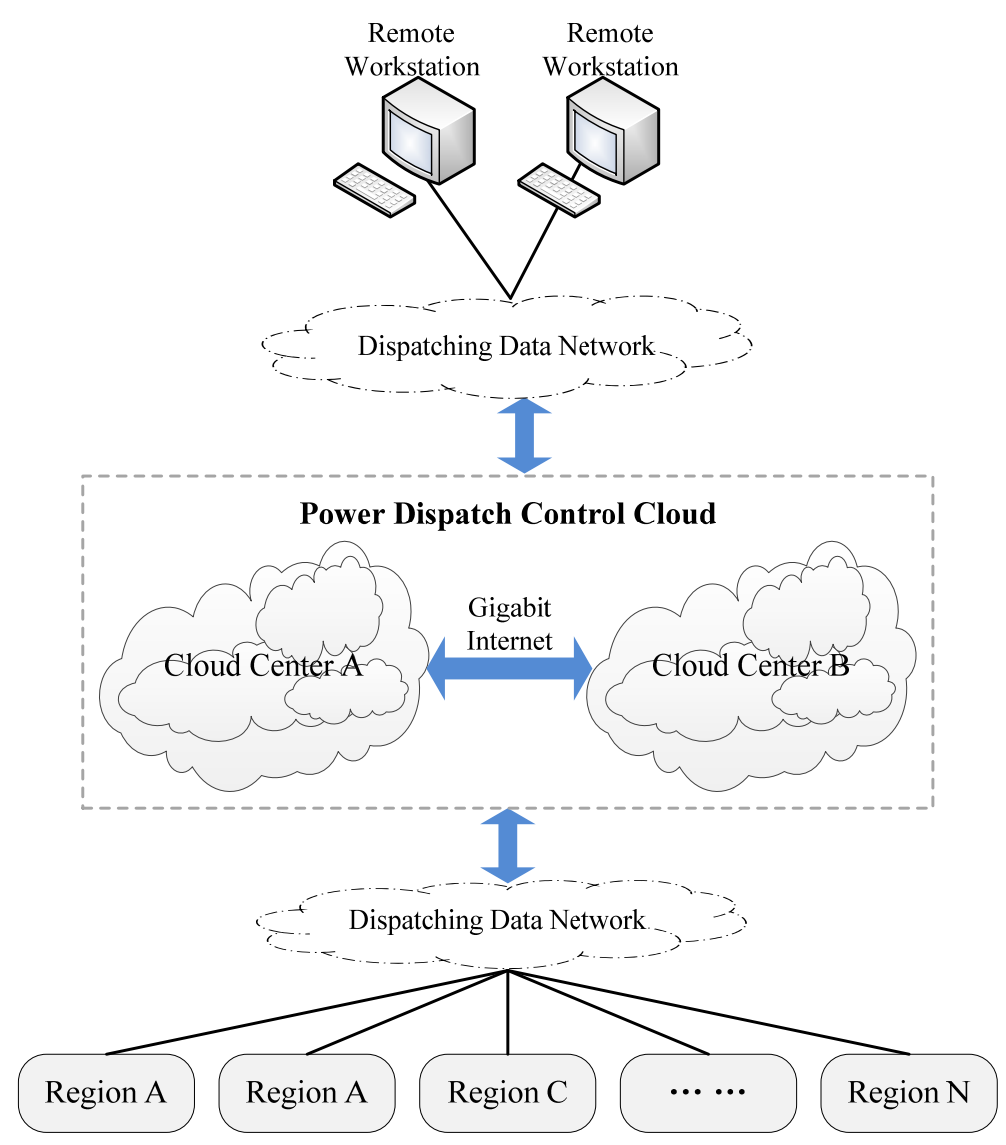

Fig. 1. The Concept of Power Dispatch Control Cloud

Eventually, application and data integration belonging to provincial dispatching institution or regional dispatching institution have been done. Two cloud centers make up an extension of local network in the storage which is connected with Gigabit Network, which provides a private interactive channel between two cloud centers.

\section{MATERIALS AND METHODS}

\section{Design of System Architecture}

Architecture of data access based on Power Dispatch Control Cloud is shown in figure 2. When the user uses the remote data access to the power of the remote data access to the internal resources of the power dispatch control cloud. Firstly, the request / response service which is deployed on the local data proxy by user is connected to the power dispatch control cloud. There when the cloud center was connected by remote data access, the proxy module could be accessed which is in the data access proxy server of the boundary of the cloud center. Of course, it can provide Load balancing, Service proxy, etc. Server can be distributed by Load balancing service in according to using condition of data access proxy server and the local or other area resources in cloud center was provided by service proxy. 

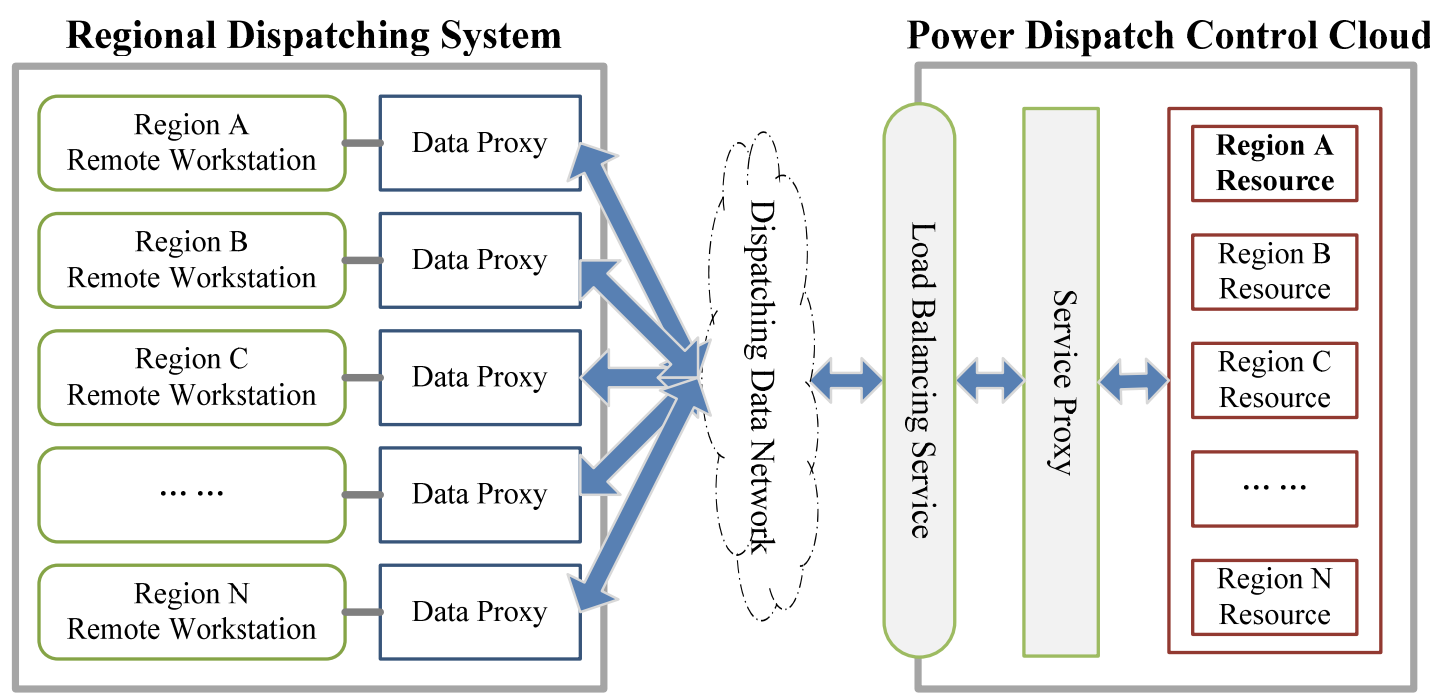

Fig. 2. Architecture of Data Access Based on Power Dispatch Control Cloud

\section{Design of Software Architecture}

To meet the functional requirements and design constraints of the cloud platform, location of each sub section of system was make clear and platform function must be easy to expand. So hierarchical structure was used in Power dispatch Control Cloud platform which consist of five parts basically. Software architecture diagram was shown in figure 3.From bottom to top, the main function of each part is as follows:

\section{Resource Layer.}

In the resource layer, the data information such as real-time data, manipulate data, model data and graph data from dispatch system of multiple regions were stored in the distributed file system, distributed database, the DM database, parallel real-time database and other database.

2. Virtualization Layer.

The virtualization layer was built on cloud computing hardware platform which include a large number of computers, storage devices and network equipment. And it is abstract of physical resources such as decentralized computing, storage, and so on. In addition, heterogeneous physical resources of cloud computing platform was dispatched uniformity and used efficiently. In a word, the virtualization layer is the key in the cloud platform.

\section{Application Support Layer.}

Combined with demand of data access mechanism, application support layer provide support for the upper application. It also support some demands of application such as Power gird operation monitoring and network analysis, etc. Moreover, basic function modules include Load Balancing, service proxy layer and other functions.

4. Application Layer.

In the application layer, the users could directly use online analysis services. For example, state estimation, network analysis, mass data processing, etc.

5. Security Layer.

Security layer is responsible for the security of cloud computing facilities and cloud platform. The main functions include identity authentication, access control, security and security audit, etc. 


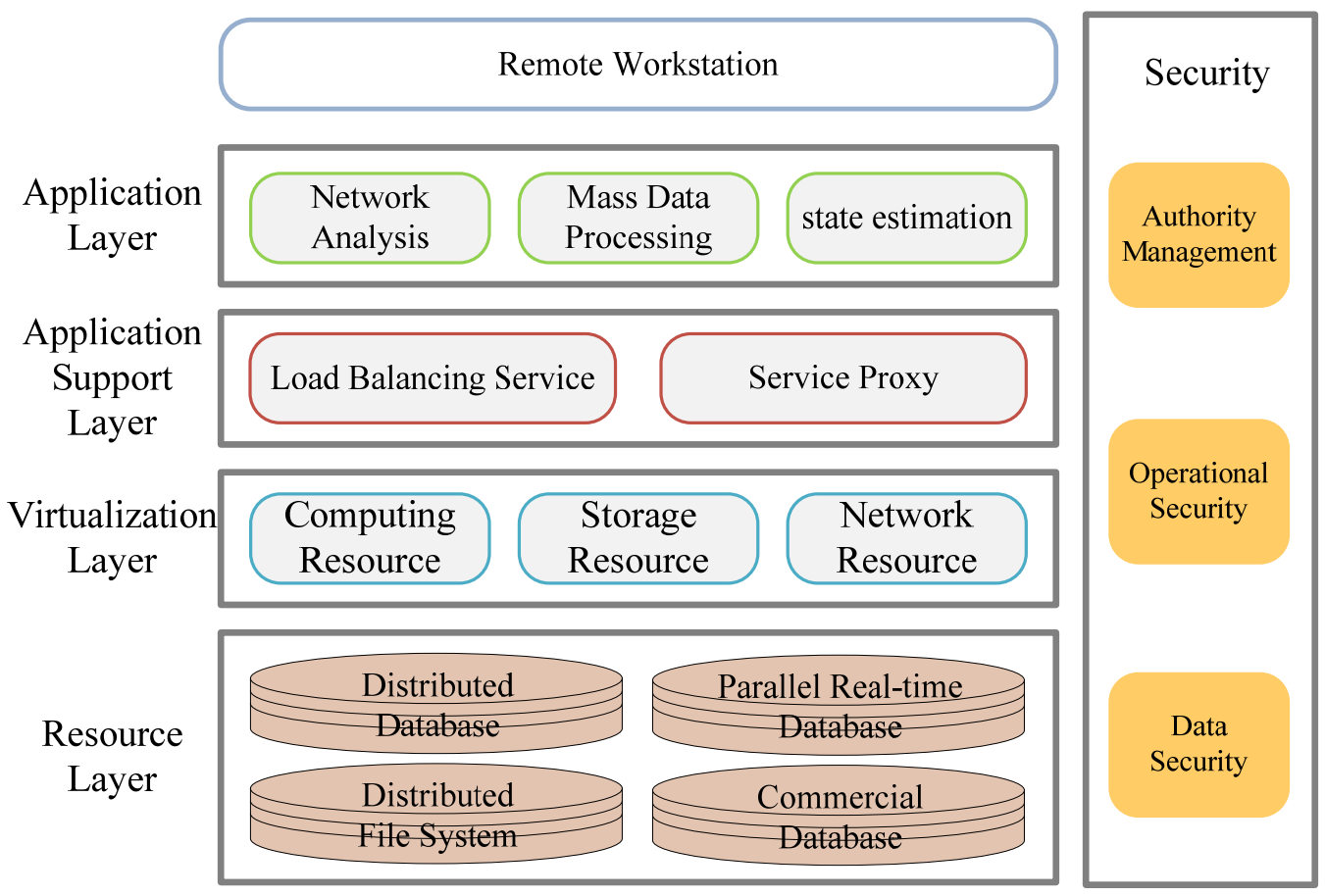

Fig. 3. Software Architecture Diagram

\section{Conclusion}

A new architecture of data access based on power dispatch control cloud is proposed in this paper to solve problem that the remote workstations from each region access Power dispatch Control Cloud for resource in the cloud center. The client-proxy-proxy-server architecture of data access ensure remote workstation accessing its own resource or resource of other regions from the cloud center with not switching the platform environment of smart grid dispatching and control system.

\section{Acknowledgement}

The authors are thankful to China State Grid Corp.

\section{References}

[1] Zhao Xiaoguang, Lan Yongping. Technolgoy of Double-Live Data Center Based on Virtualization [J] .Information Technology and Informatization, 2014, 05:270-271+273.

[2] WU Li-le. Design and application of cloud computing data center based on double-live disaster tolerance storage technology. Electronic Design Engineering [J], 2015, 06:190-192.

[3] Wang Dewen. Power data center infrastructure and its key technology based on cloud computing [J].Automation of Electric Power Systems, 2012, 11:67-71+107.

[4] Chen Ming. Research and implementation of key technologies of "dispatch cloud" in power system [D]. Guangxi University, 2013.

[5] MU Lianshun, CUI Lizhong, AN Ning. Research and Practice of Cloud Computing Center for Power System [J]. Power System Technology, 2011, 06:171-175.

[6] Yang Xuxin Liu Junyong Ji Hongliang et al. Cloud computing of power system [J].Sichuan Electric Power Technology, 2010,03:71-76.

[7] Wang Lisai, Yang Mingyu, Sun Yueqin, Wang Dong, Zhang Yonghao. Research review of power cloud [J]. Electric Power Information Technology, 2011, 05:20-23. 
[8] Mao Yufeng. Design and Implementation of Massive Power Data Analyzing System Based on Cloud Computing [D]. Beijing Jiaotong University, 2015

[9] Pang Songtao. Dispatching research of resource planning in power cloud based on data mining [A]. 2014 electric power industry information technology [C]. China Electric Power Engineering Institute of electric information technology professional committee: 2014:1.

[10] Li Hailong. Research and application of data mining algorithm in data analysis platform of power cloud [D]. North China Electric Power University, 2014

[11] WU Kaifeng, LIU Wantao, LI Yanhu et al. Analytical techniques and application using power big data based on cloud computing [J]. Electric Power, 2015, 02:111-116+127.

[12] Ma Yumei. Research and application of data integration technology in power system [D]. North China Electric Power University (Hebei), 2006. 